\title{
Primidone in essential tremor of the hands and head: a double blind controlled clinical study
}

\author{
LESLIE J FINDLEY,${ }^{*}$ LYNN CLEEVES, $†$ STEPHANO CALZETTI
}

From the Regional Centre for Neurology and Neurosurgery, Oldchurch Hospital, Romford, Essex, ${ }^{*}$ MRC Neuro-Otology Unit, National Hospital, London UK† and Instituto di Clinica Neurologica, Universita di Parma, Parma, Italy $\ddagger$

SUMMARY Uncontrolled clinical studies have suggested that primidone may be effective in reducing essential tremor thus providing a valuable alternative to beta-adrenoreceptor antagonists which are currently the drugs of first choice. A double blind, placebo controlled trial of primidone in essential tremor of the hands and head was carried out using both clinical and objective methods of assessment. Primidone was significantly superior to placebo in reducing the magnitude of hand tremor, its efficacy being comparable to that of propranolol. In two patients tremor was reduced to non-symptomatic levels, an effect rarely seen with propranolol. No consistent attenuation of head tremor was found. There was no correlation between serum primidone or derived phenobarbitone concentrations and the reduction of hand tremor. An acute toxic reaction to an initial small dose $(62.5 \mathrm{mg})$ of primidone was seen in six of 22 patients.

Familial or essential tremor is a common disorder characterised by tremor during the maintenance of posture and active movement. ${ }^{.}$Essential tremor can affect any part of the body, but most commonly the hands. Head tremor can occur in combination with hand tremor or, more rarely, in isolation.

The drug treatment of essential tremor remain poor and often unsatisfactory. The betaadrenoreceptor antagonist propranolol, currently the drug of choice in this condition, benefits only about two-thirds of the patients treated and the clinical response is variable and usually incomplete..$^{2.3}$ Furthermore a number of contra-indications restricts the use of this class of drug, particularly in the elderly.

It has been stated that head tremor is more resistant to treatment than tremor of the hands, ${ }^{45}$ and this accords with our clinical experience. However the results of a recent controlled study suggest that head and hand tremor may respond similarly to propranolol. ${ }^{6}$

In recent years it has been reported that primidone, a well established anticonvulsant, can be

Address for reprint requests: Dr LJ Findley, National Hospital for Nervous Diseases, Queen Square, London WC1N 3BG, UK

Received 11 January 1985

Accepted 2 February 1985 of value in the treatment of essential tremor. ${ }^{78}$ However, these studies were not conducted under controlled conditions with objective methods of assessment, and thus do not provide a reliable and quantitative indication of the tremorlytic efficacy of the drug in these patients. Furthermore, the spectrum of efficacy of primidone remains undefined, that is, whether its effect is restricted to tremor of the hands or whether tremor in other body parts (for example head) can be similarly attenuated. Nor has it been established whether a therapeutic range of blood concentration of the parent drug or derived phenobarbitone can be correlated with the clinical response.

In a previous short report ${ }^{9}$ we presented the preliminary results of a controlled quantitative study of primidone in 11 patients with essential tremor of the hands. In the present paper we report the results of a more extensive study using both clinical and objective methods of evaluation, in 22 patients with essential tremor of hands 14 of whom also had tremor of the head.

\section{Patients and methods}

Twenty-two patients (15 male, seven female) with moderate to severe essential tremor, aged 16-82 years (median age 59 years), were included in the study. The diagnosis was established on the basis of chronic monosyptomatic postural tremor affecting the hands and/or the head, and 911 
by means of ancillary laboratory investigations where necessary. In eight patients the tremor involved only the hands, in 14 both the hands and the head were involved. All patients had been symptomatic for at least one year (median duration 10 years, range $1-50$ years) and eight had a positive family history of tremor affecting the hands and/or head. Patients with a history of excessive alcohol consumption were excluded. Eighteen of the patients had not received previous medication for tremor; four patients who had been on chronic therapy with betaadrenoreceptor antagonists discontinued their treatment at least 2 weeks before entering the study.

The study was double blind and placebo controlled. Primidone and placebo were given in randomised order according to a cross-over design for a period of five weeks each. Since a previous open pilot study with primidone commencing at $125 \mathrm{mg}$ daily revealed an unacceptable incidence of side effects in more than $50 \%$ of patients, primidone was started at a dosage of $62.5 \mathrm{mg}$ daily (1/4 standard tablet) and increased by $62.5 \mathrm{mg}$ daily up to a maximum of $750 \mathrm{mg}$ daily or until side-effects necessitated reduction in dosage. Both primidone and placebo were given in three divided daily doses.

Tremor was assessed at the end of each five week period, at a time when steady state level of serum primidone and phenobarbitone had been reached. Patients were instructed to take their last tablet 3 hours prior to tremor assessment and to abstain from taking alcohol, tobacco or caffeinated beverages for 12 hours beforehand. On each occasion three separate one minute samples of tremor were recorded from the hands maintained extened in a pronated attitude with the forearm supported up to the wrist. The hands were allowed to relax freely between recordings. Hand tremor was measured by miniature DC-monoaxial linear piezoresistive accelerometers taped to the dorsum of each hand, in the second interspace, $1 \mathrm{~cm}$ proximal to the metacarpophalangeal joints, with their sensitive axes oriented in the vertical plane. Similarly, two one-minute samples of tremor were recorded from the head, with the patient seated in an armchair looking straight ahead. Two accelerometers were taped to the forehead, one with its sensitive axis oriented in the sagittal (A-P) plane and the other in the lateral (G-D) plane, so that both components of head tremor were measured simultaneously.

The recordings were quantified as the average frequency spectrum of $150,10.24 \mathrm{~s}$, overlapping samples taken equally from each of the tremor recordings, according to a method previously described. ${ }^{3}$ Measurements were taken of the frequency of the dominant peak of tremor and its magnitude calibrated in units of root-mean-square acceleration $\left(\mathrm{rms} g\right.$, where $\left.1 \mathrm{~g}=981 \mathrm{~cm} / \mathrm{s}^{2}\right)$. Since it was found that the dominant peak frequency of tremor did not vary significantly either within or between recordings, the magnitude of tremor acceleration in each condition is proportional to displacement according to the relationship:

$\underset{\operatorname{rms}(\mathrm{cm})}{\text { Tremor amplitude }}=\frac{\text { magnitude of acceleration }}{4 \pi^{2} \times \text { frequency }^{2}} \times 981$

Additional evaluation of tremor was performed by blind clinical assessment of postural tremor of the hands and of the head, by performance tests (handwriting, drawing geometrical figures and tracing into a Archimedes spiral) and by patients' self assessment of their abilities in daily activities along with the observations of family and friends.

Clinical assessment, self assessment and performance tests were scored according to an arbitrary scale from zero (asymptomatic, no impairment) to 5 (severe tremor, maximum impairment). Performance tests were rated, blind, by three different assessors and the final score for each patient was obtained from the mean of those ratings.

At the completion of tremor assessment, venous blood samples were taken for determination of serum primidone and phenobarbitone levels by enzyme-mediated immunoassay. ${ }^{10}$

Only data from the more involved hand and from the main component of head tremor (during placebo regimen) were used for statistical analysis. Changes in tremor magnitude, performance tests, clinical and self rating were analysed using the Wilcoxon test for paired differences. The relationship between responses to primidone and some clinical variables (age of patient, positive family history, duration of symptoms, magnitude and peak frequency of tremor, and serum concentration of primidone and phenobarbitone) was computed by Spearman's rank correlation coefficient.

\section{Results}

Only 16 patients completed the study. Five patients were withdrawn as a result of unacceptable side effects (see below). A sixth patient was excluded after admitting to having taken alcohol prior to his visits to the laboratory. Of the patients who completed the study four were unable to tolerate the maximum dosage of primidone $(750 \mathrm{mg})$ and received a daily dosage of between 125 and $500 \mathrm{mg}$. Two patients received both primidone and placebo for a two week period only.

The dominant peak frequency of tremor in the more involved hand ranged between 4.2 and $7 \cdot 0 \mathrm{~Hz}$. There was no significant difference in the dominant peak frequency of hand tremor whilst treated with either primidone or placebo.

Mean magnitude of hand tremor during placebo regimen was 96.6 milli-g (range 11.0 to 675 milli-g or $0.008-0.62 \mathrm{~cm}$ displacement). Primidone was found to be significantly superior to placebo in reducing the magnitude of hand tremor when its effect was calculated both as absolute change in units of acceleration $(p<0.01)$ and percentage change against placebo $(p<0 \cdot 01)$. The mean percentage reduction of tremor magnitude produced by primidone compared to placebo was $55.9 \%( \pm 7.6$ SEM). Fifteen patients had lower tremor magnitude on primidone than placebo (fig 1). One patient showed a small increase in tremor magnitude on primidone. In two severely affected patients, primidone suppressed tremor to non-symptomatic 


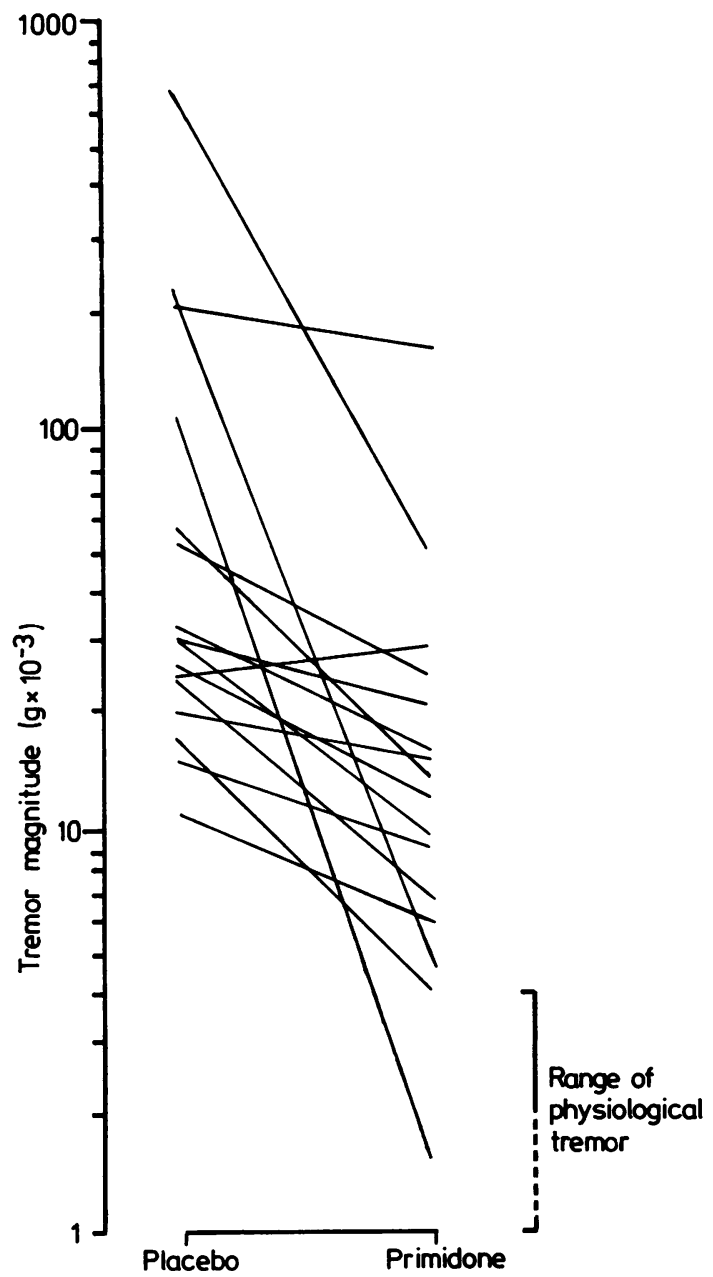

Fig 1 Values of tremor magnitude (in $\mathrm{g} \times 10^{-3}$ ) for individual patients after placebo and primidone.

levels, that is within the range of physiological tremor. Primidone was also found to be significantly superior to placebo on the basis of performance tests $(p<0.01)$, clinical assessment $(p<0.02)$ and patient's self assessment $(p<0.01)$ (fig 2$)$.

There was no correlation between the response to primidone (expressed either as percentage or absolute change) and age of patient, duration of symptoms, family history, peak frequency and magnitude of hand tremor during placebo.

Nine patients with tremor of the head completed the study but accelerometric recordings were available for only five of these. All nine patients were rated clinically and by self-assessment. Head tremor was more severe in the lateral plane ("no-no" tre- mor) in three patients and in the antero-posterior direction ("yes-yes" tremor) in two.

The frequency of the main component of head tremor in these patients ranged from $2 \cdot 3-6 \cdot 2 \mathrm{~Hz}$. The dominant peak frequency of head tremor was similar in both primidone and placebo treatments.

Magnitude of head tremor during placebo regimen ranged from 1.9 to 21.3 milli-g rms (mean 8.5 milli- $g$ ). On the basis of accelerometric measures primidone was not consistently superior to placebo in reducing the magnitude of head tremor. However, response to primidone in this small group of patients exhibited extreme variability (mean 6.9\%) ranging from a decrease of $75.3 \%$ to an increase of $63.2 \%$. In the same patients hand tremor showed a consistent reduction (mean $72.0 \%$, range 52.6 to $98.6 \%)$. In agreement with the results obtained by
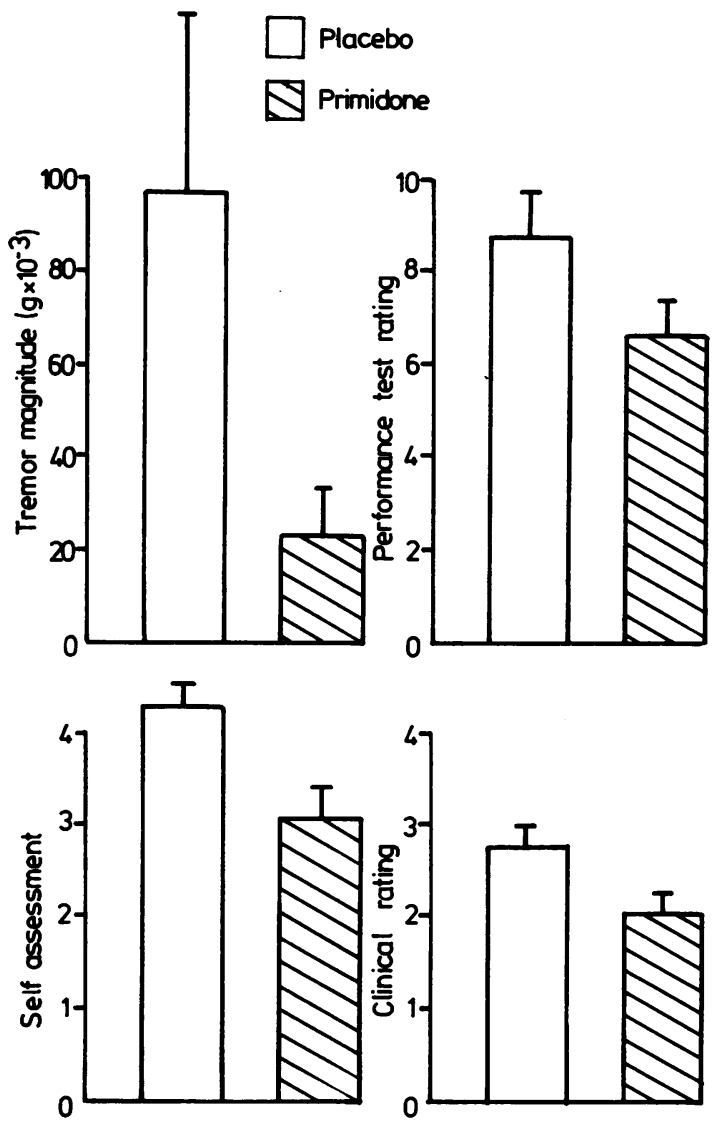

Fig 2 Mean (+1 SEM) values of hand tremor magnitude for 16 patients after placebo and primidone as assessed by objective measurement $(p<0.01)$, performance tests $(p<$ $0.01)$, patient's self rating $(p<0.01)$ and clinical rating $(p<0.02)$. 
the accelerometric method, clinical evaluation showed no difference for primidone and placebo. However, according to patients' self-assessment, all but one obtained a lower score on primidone than on placebo.

Serum primidone levels ranged from 18 to 100 $\mu \mathrm{mol} / \mathrm{l}$ (median $48 \mu \mathrm{mol} / \mathrm{l}$ ) and serum phenobarbitone concentrations from 5 to $87 \mu \mathrm{mol} / \mathrm{l}$ (median $55 \mu \mathrm{mol} / \mathrm{l}$ ). There was no correlation between the absolute or percentage reduction in hand tremor magnitude produced by primidone and either serum primidone or phenobarbitone concentrations.

In five patients ( 4 male, 1 female; age range 52$68 \mathrm{yr}$ ), an acute toxic reaction occurred after the first $62.5 \mathrm{mg}$ dose of primidone necessitating termination of treatment and withdrawal from the study. This reaction consisted of nausea, vomiting, ataxia and giddiness, so severe as to force the patient to bed. The duration of these symptoms varied from 12 to 72 hours.

Less severe dose-related side effects occurred in 11 patients on primidone and two patients on placebo. These effects consisted of a variable combination of sedation, day-time sleepiness, tiredness and depression which were attenuated with a reduction of primidone dosage.

\section{Discussion}

The results of this study confirm and extend our preliminary report ${ }^{9}$ and are in agreement with previous findings showing primidone to be effective in reducing hand tremor in patients with essential tremor..$^{8}$ Of the patients studied, all but one (94\%) responded to the drug (response being defined as a tremor reduction of at least $15 \%$ of placebo values). The efficacy of the drug was demonstrated by both objective and subjective methods of assessment. Primidone showed a degree of efficacy at least comparable to that reported following propranolol at a dosage of $240 \mathrm{mg}$ daily. ${ }^{3}$ In two severely affected patients primidone reduced tremor magnitude to within the range of non-symptomatic physiological tremor, an effect only rarely observed with propranolol or other beta-adrenoreceptor antagonists in these patients. "1

The lack of correlation between the response to primidone and the serum concentrations of the parent drug and derived phenobarbitone may indicate that individual susceptibility to primidone and/or its metabolites is the main determinant of tremorlytic efficacy. However, it cannot be excluded that at doses and/or blood concentrations of primidone higher that those used in this study, some correlation between the clinical response and drug serum concentration could become evident.

In contrast to a previous, uncontrolled trial, ${ }^{8}$ primidone did not prove consistently superior to placebo in reducing head tremor magnitude in patients with essential tremor, on the basis of both objective and clinical evaluation. The differential response of hand and head tremor to primidone, as demonstrated in this study, is in accordance with our experience with propranolol and suggests that different pathophysiological mechanisms may underly tremors affecting different body parts in the same patient. Anecdotally, we have found that, in some patients, primidone and propranolol in combination, can attenuate head tremor when neither drug alone was effective.

The mode of action of primidone in essential tremor is unknown and it is still not clear whether the tremorlytic effect is mediated by the parent drug itself and/or by derived active metabolites, that is phenobarbitone and phenylethylmalonamide (PEMA). Although in an earlier report is was suggested that the clinical efficacy is mainly related to PEMA, ${ }^{7}$ a later, controlled study ${ }^{12}$ failed to demonstrate any significant effect of this metabolite on tremor. More recently Baruzzi et $a^{13}$ have shown that phenobarbitone can be of value in essential tremor. Even though these authors reported a large placebo effect, the response to phenobarbitone was significantly greater. The results of Baruzzi et $a^{13}$ are $\stackrel{\mathbb{Q}}{\vec{Q}} \vec{c}$ not directly comparable to the results of the present $\frac{\infty}{\infty}$ study. However, although similar blood concentra- $<\frac{0}{\mathbb{D}}$ tions of phenobarbitone were observed in the twoo studies, a greater reduction in tremor was found in the present study, which suggests that the parent drug itself contributes, to some extent, to the overall tremorlytic effect. The relative efficacy of primidone and phenobarbitone has not yet been evaluated systematically in the same population of patients.

The high incidence of acute toxic reactions following the initial, small oral dose of primidone (for example $62.5 \mathrm{mg}$ ) in patients with essential tremor ( $22 \%$ in the present study) has been previously reported by O'Brien et al.? The reason for this high incidence of "early" toxicity in patients with essential tremor is unclear. The latency of onset of symptoms was such that no significant metabolism of the drug could have occurred. It has been suggested that such side effects are due to delayed breakdown of the parent drug into its metabolites because of the absence of hepatic enzyme induction in patients previously unexposed to other anticonvulsants. ${ }^{14}$ This suggestion is indirectly supported by the lower incidence of acute reactions following primidone in epileptic patients, most of whom have previously received other anticonvulsant drugs.$^{14}$ Furthermore, neither PEMA nor phenobarbitone has been reported to produce any acute adverse reaction when first given to patients with essential tremor. ${ }^{12}{ }^{13}$ 
In order to reduce the incidence and severity of the initial acute toxicity of the drug it may be beneficial to give a short pre-treatment course of an hepatic enzyme-inducing drug, namely phenobarbitone before starting primidone treatment with a slowly incremental dose regimen (for example at $62.5 \mathrm{mg}$ for the first 2-3 days).

This study has shown that primidone can be effective in suppressing essential tremor of the hands and its effect would seem to be at least comparable to that of propranolol at a dose of $240 \mathrm{mg}$ daily. In two patients with large amplitude tremor, suppression was complete, a phenomenon not seen in patients on beta-adrenoreceptor blocking drugs. It therefore provides an alternative form of therapy in patients symptomatic enough to require chronic treatment. It should however, be emphasised that one in five patients has shown acute "toxicity" on very small doses of primidone. Although this side effect is short-lived, it can be very severe. Thus, all patients must be warned of this possibility and one could argue that elderly patients should be admitted to hospital for initiation of therapy.

Open follow-up studies have shown that patients can maintain their response for more than two years. However, further studies are needed to monitor long-term efficacy. Controlled, comparative studies of primidone and phenobarbitone are needed to evaluate their relative tremorlytic effects.

\section{References}

' Findley LJ. Essential tremor: introductory remarks. In: Findley LJ, Capildeo R, eds. Movement DisordersTremor. London: Macmillan Press, 1984:207-10.

${ }^{2}$ Larsen TA, Calne DB. Essential tremor. Clin Neuropharmacol 1983;6:185-206.
${ }^{3}$ Calzetti S, Findley LJ, Perucca E, Richens A. The response of essential tremor to propranolol: evaluation of clinical variables governing its efficacy on prolonged administration. J Neurol Neurosurg Psychiatry 1983;46:393-8.

${ }^{4}$ Murray TJ. Treatment of essential tremor with propranolol. Can Med Assoc J 1972;107:984-6.

5 Young RR, Shahani BT. Pharmacology of tremor. In: Klawans HL, ed. Clinical Neuropharmacology, vol 4. New York: Raven Press, 1979:139-56.

- Koller WC. Propranolol therapy for essential tremor of the head. Neurology (NY) 1984;34:1077-9.

7 O'Brien MD, Upton AR, Toseland PA. Benign familial tremor treated with primidone. $\mathrm{Br} \mathrm{Med} J$ 1981;282:178-80.

${ }^{8}$ Chakrabarti A, Pearce JMS. Essential tremor: response to primidone. J Neurol Neurosurg Psychiatry 1981;44:650.

${ }^{9}$ Findley LJ, Calzetti S. Double-blind controlled study of primidone in essential tremor: preliminary results. $\mathrm{Br}$ Med J 1982;285:608.

${ }^{10}$ Sun L, Walwick ER. Primidone analyses: correlation of gas chromatographic assay with enzyme immunoassay. Clin Chem 1976;22:901-2.

" Cleeves LA, Findley LJ. Beta-adrenoreceptor mechanisms in essential tremor: a comparative single dose study of an effect of a non-selective and a beta- 2 selective adrenoreceptor antagonist. $J$ Neurol Neurosurg Psychiatry 1984;47:976-82.

12 Calzetti S, Findley LJ, Pisani F, Richens A. Phenylethylmalonamide in essential tremor. A double-blind controlled study. J Neurol Neurosurg Psychiatry 1981;44:932-4.

${ }^{13}$ Baruzzi A, Procaccianti G, Martinelli P, et al. Phenobarbital and propranolol in essential tremor: a doubleblind controlled clinical trial. Neurology (NY) 1983;33:296-300.

${ }^{14}$ Feely M. Benign familial tremor treated with primidone. Br Med J 1981;282:740-1. 